\title{
REMARKS ON THE COMMUTATIVITY OF THE RADICALS OF GROUP ALGEBRAS
}

\author{
by SHIGEO KOSHITANI
}

(Received 15 August, 1977)

Let $K$ be an arbitrary field with characteristic $p>0, G$ a finite group of order $p^{a} g^{\prime}$ with $\left(p, g^{\prime}\right)=1, P$ a $p$-Sylow subgroup of $G$ and $G^{\prime}$ the commutator subgroup of $G$. For a ring $R$ denote by $J(R)$ the Jacobson radical of $R$ and by $Z(R)$ the centre of $R$. We write $K G$ for the group algebra of $G$ over $K$.

On the commutativity of $J(K G)$ there are works of D. A. R. Wallace [11] and W. Hamernik [3]. The first aim of this paper is to investigate the structure of $G$ when $J(K G)$ is commutative. Our result can be stated as follows: if $J(K G)$ is commutative and $J(K G)^{2} \neq 0$, then $N_{G}(P)=C_{G}(P)$ and $N_{G}(P)$ is abelian, where $N_{G}(P)$ and $C_{G}(P)$ are the normalizer of $P$ in $G$ and the centralizer of $P$ in $G$, respectively.

D. A. R. Wallace [11] and W. Hamernik [3] obtained a necessary and sufficient condition on $G$ for $J(K G)$ to be commutative when $p$ is odd. Indeed, they proved that when $p$ is odd and when $G$ is a nonabelian group of order divisible by $p, J(K G)$ is commutative if and only if $G^{\prime} P$ is a Frobenius group with complement $P$ with kernel $G^{\prime}$. So in the present paper we shall obtain a necessary and sufficient condition of $G$ for $J(K G)$ to be commutative for any prime number $p$ not necessarily odd. That is to say, we shall prove that $J(K G)$ is commutative if and only if $G$ is a group of the following two types: (i) $|G|$ is not divisible by $2^{2}$ when $p=2$, and $|G|$ is not divisible by $p$ when $p$ is odd; (ii) $G$ is a $p$-nilpotent group with an abelian $p$-Sylow subgroup $P, b_{0}=\left|O_{p^{\prime}}(G): G^{\prime}\right|$, $b_{1}=\ldots=b_{a-2}=0$, and if $p$ is odd, $b_{a-1}=0$, where $b_{k}$ is the number of $p$-regular conjugate classes $K_{j}$ of $G$ such that the number of elements of $K_{j}$ is divisible by $p^{k}$ and not by $p^{k+1}$ for $k=0, \ldots, a$. By [11, Theorem 1] and [3, Corollary 5.2], when $p$ is odd, $J(K G)$ is commutative if and only if $J(K G) \subseteq Z(K G)$. But when $p=2$, this does not hold in general.

Throughout this paper we shall use the following notations. Denote by $[V: K]$ the $K$-dimension of a $K$-vector space $V$. If $S$ is a subset of $G,|S|$ will denote the number of elements of $S, N_{G}(S)$ and $C_{G}(S)$ will denote the normalizer of $S$ in $G$ and the centralizer of $S$ in $G$, respectively, and let $\hat{S}=\sum_{s \in S} s$ in $K G$ when $S \neq \varnothing$ and let $\hat{S}=0$ in $K G$ when $S=\varnothing$. For a positive integer $t$ and a ring $R$ we write $R_{t}$ for the ring of all $t \times t$ matrices with entries in $R$.

To begin with we shall study $G$ when $J(K G)$ is commutative.

Theorem 1. Suppose that $|G|$ is divisible by $2^{2}$ when $p=2$ and that $|G|$ is divisible by $p$ when $p$ is odd. If $J(K G)$ is commutative, then $N_{G}(P)=C_{G}(P)$ and $N_{G}(P)$ is abelian, where $P$ is a $p$-Sylow subgroup of $G$.

Proof. Since $K G / J(K G)$ is a separable $K$-algebra (cf. [4, Proposition 12.11]), $J(E G)=$ $E \otimes_{K} J(K G)$ for any extension field $E$ of $K$. So we may assume that $K$ is algebraically

Glasgow Math. J. 20 (1979) 63-68. 
closed. Let $1=\sum_{i=1}^{n} \sum_{j=1}^{f_{i}} e_{i j}$ be a decomposition of the unit element of $K G$ into a sum of mutually orthogonal primitive idempotents of $K G$ such that $K G e_{i j} \cong K G e_{i^{\prime} j^{\prime}}$ if and only if $i=i^{\prime}$. Set $e_{i}=e_{i 1}, U_{i}=K G e_{i}, F_{i}=U_{i} J J(K G) U_{i}$ and $u_{i}=\left[U_{i}: K\right]$, and hence $f_{i}=\left[F_{i}: K\right]$ for each $i$. Let $F_{1}$ be the trivial $K G$-module, and so $f_{1}=1$.

By [11, Theorem 2], $G$ is $p$-nilpotent and $P$ is abelian. So $K G$ is primary decomposable from [6, Theorem 1]. Thus each block of $K G$ contains, up to isomorphism, only one irreducible $K G$-module. Put $B_{i}=\sum_{j=1}^{f_{i}} \oplus K G e_{i j}$ for each $i . B_{1}, \ldots, B_{n}$ are all blocks of $K G$. Set $H=O_{p^{\prime}}(G)$, the largest normal subgroup of $G$ of order prime to $p$. Since $G$ is $p$-nilpotent, it follows from [5, Theorems 2,7] that

$$
B_{i} \cong K H e_{i 1}^{\prime} \otimes_{K} K^{c} P_{i} \otimes_{K} K_{i} \text {, as } K \text {-algebras, }
$$

where $e_{i 1}^{\prime}$ is a centrally primitive idempotent of $K H, G_{i}=\left\{x \in G \mid x^{-1} e_{i 1}^{\prime} x=e_{i 1}^{\prime}\right\}, t_{i}=$ $\left|G: G_{i}\right|, P_{i}$ is a $p$-Sylow subgroup of $G_{i}$ and $K^{c} P_{i}$ is a twisted group ring of $P_{i}$ over $K$ with respect to the factor set $c$ for each $i=1, \ldots, n$. Since $K$ is an algebraically closed field with characteristic $p>0$ and $P_{i}$ is a $p$-group, $K^{c} P_{i} \cong K P_{i}$ as $K$-algebras for each $i$ (cf. [7, Lemma 2.1]). Hence

$$
B_{i} \cong K H e_{i 1}^{\prime} \otimes_{K} K P_{i} \otimes_{K} K_{4}, \text { as } K \text {-algebras. }
$$

Put $h_{i}^{2}=\left[K H e_{i 1}^{\prime}: K\right]$ and $h_{i}>0$. By [6, Theorem 3], $f_{i}=h_{i} t_{i}$. This shows that $B_{i} \cong\left(K P_{i}\right)_{f_{1}}$ as $K$-algebras and that

$$
J\left(B_{i}\right) \cong\left(J\left(K P_{i}\right)\right)_{f_{i}}
$$

for each $i$. Now, let us divide $B_{1}, \ldots, B_{n}$ into the following three types:

(a) $J\left(B_{i}\right)=0$.

(b) $J\left(B_{i}\right) \neq 0, J\left(B_{i}\right)^{2}=0$.

(c) $J\left(B_{i}\right)^{2} \neq 0$.

When $B_{i}$ is of type $(a)$ or $(b), f_{i}$ is divisible by $p$. Indeed, if $B_{i}$ is of type $(a), u_{i}=f_{i}$ and so $p^{a}$ divides $f_{i}$ from [1, (18)]. If $B_{i}$ is of type $(b)$, by [11, Lemma 7], $p=2$ and $u_{i}=2 f_{i}$, and so $f_{i}$ is divisible by 2 since $a \geqq 2$ and $2^{a}$ divides $u_{i}$ from $[1,(18)]$. Hence the principal block $B_{1}$ is of type $(c)$. By rearranging the numbers $2, \ldots, n$, we may assume that $B_{1}, \ldots, B_{m}$ are of type $(c)$ and that $B_{m+1}, \ldots, B_{n}$ are of type $(a)$ or (b) for some $m \leqq n$. If $B_{i}$ is of type (c), since $J(K G)$ is commutative, it follows from $\left(^{*}\right)$ that $f_{i}=1$ and so $h_{i}=t_{i}=1$. This implies that $B_{1}, \ldots, B_{m}$ are all blocks of $K G$ with defect $a$.

Next, since $P$ is an abelian $p$-Sylow subgroup of $G$ and $G$ is $p$-nilpotent, by $[5, \S 3$ (p. 184)], $N_{G}(P)=C_{G}(P)$. Set $N=N_{G}(P)$ and $\tilde{H}=H \cap N=O_{p^{\prime}}(N)$. Since $N$ is $p$ nilpotent, it follows from [6, Theorem 1] and [5, Lemma 2] that $m$ is equal to the number of blocks of $K N$. Let $1=\sum_{i=1}^{m} \sum_{j=1}^{f_{i}} \bar{e}_{i j}$ be a decomposition of the unit element of $K N$ into a sum of mutually orthogonal primitive idempotents of $K N$ such that $K N \tilde{e}_{i j} \cong K N \tilde{e}_{i^{\prime} j^{\prime}}$ if and only if $i=i^{\prime}$. Put $\tilde{e}_{i}=\tilde{e}_{i 1}, \tilde{U}_{i}=K N \tilde{e}_{i}, \tilde{F}_{i}=\tilde{U}_{i} / J(K N) \tilde{U}_{i}$ and $\tilde{u}_{i}=\left[\tilde{U}_{i}: K\right]$, and hence 
$\tilde{f}_{i}=\left[\tilde{F}_{i}: K\right]$ for each $i$. Set $\tilde{B}_{i}=\sum_{i=1}^{f_{H}} \oplus K N \tilde{e}_{i j}$ for each $i$, and so $\tilde{B}_{1}, \ldots, \tilde{B}_{m}$ are all blocks of $K N$. Since $N$ is $p$-nilpotent, as for $B_{i}$, we can write

$$
\tilde{B}_{i} \cong K \tilde{H} \tilde{e}_{i 1}^{\prime} \otimes_{K} K \tilde{P}_{i} \otimes_{K} K_{\mathrm{h}} \text {, as } K \text {-algebras, }
$$

where $\tilde{e}_{i 1}^{\prime}$ is a centrally primitive idempotent of $K \tilde{H}, \tilde{G}_{i}=\left\{y \in N \mid y^{-1} \tilde{e}_{i 1}^{\prime} y=\tilde{e}_{i 1}^{\prime}\right\}, t_{i}=$ $\left|N: \tilde{G}_{i}\right|$ and $\tilde{P}_{i}$ is a $p$-Sylow subgroup of $\tilde{G}_{i}$ for each $i=1, \ldots, m$. Since $P$ is normal in $N$, all blocks of $K N$ have defect $a$. Put $\tilde{h}_{i}^{2}=\left[K \tilde{H} \tilde{e}_{i 1}^{\prime}: K\right]$ and $\tilde{h}_{i}>0$. By [6, Theorem 3], $\tilde{f}_{i}=\tilde{h}_{i} \tilde{t}_{i}$. Hence $\tilde{t}_{i}$ is not divisible by $p$ and this shows that $\tilde{t}_{i}=1$ for all $i$. This implies that $\tilde{e}_{i 1}^{\prime}$ is a centrally primitive idempotent of $K N$ and that $\bar{P}_{i}=\boldsymbol{P}$ for all $i$.

By rearranging the numbers $1, \ldots, m$, we can assume that $B_{i}$ corresponds to $\tilde{B}_{i}$ through the Brauer homomorphism for each $i=1, \ldots, m$ (cf. [2, Lemma 56.1, Theorem 58.3 (Brauer's first main theorem)]). Fix any $i$ such that $1 \leqq i \leqq m$. Since $t_{i}=1, e_{i 1}^{\prime}$ is a centrally primitive idempotent of $K G$, and so we may write $e_{i 1}^{\prime}=e_{i}$ since $f_{i}=1$. Put $B=B_{i}$, $e=e_{i 1}^{\prime}=e_{i}, \tilde{B}=\tilde{B}_{i}$ and $\tilde{e}=\tilde{e}_{i 1}^{\prime}$. Let $\left\{K_{r}\right\}$ be the set of all conjugate classes of $G$. The Brauer homomorphism $\sigma: Z(K G) \rightarrow Z(K N)$ is defined as $\sigma \widehat{\left(K_{r}\right)}=\widehat{K_{r} \cap N}$ for each $r$. We know that $\sigma(e)=\tilde{e}$. $e$ is a centrally primitive idempotent of $K H$ and $\tilde{e}$ is a centrally primitive idempotent of $K \tilde{H}$. Thus, if we let $\left\{L_{t}\right\}$ be the set of all conjugate classes of $H$ and if we define $\sigma^{\prime}: Z(K H) \rightarrow Z(K \tilde{H})$ as $\sigma^{\prime}\left(\overline{L_{t}}\right)=\overline{L_{t} \cap N}$ for each $t$, it follows that $\sigma^{\prime}(e)=\tilde{e}$. On the other hand, $[K H e: K]=1$, and so $K H e=K e$. Take any $h \in \tilde{H}$. We shall claim that $h \tilde{e} \in K \tilde{e}$. Since $K H e=K e$, we can write he $=\delta e$ for some $\delta \in K$. Let $e=\sum_{t} \alpha_{t} . \widehat{L}_{t}$, where $\alpha_{t} \in K$. Thus, $\tilde{e}=\sigma^{\prime}(e)=\sum_{t} \alpha_{t} \cdot\left(\widehat{L_{t} \cap N}\right)$. Since $\sum_{t} \alpha_{t} \cdot h \widehat{L}_{t}=\sum_{t} \delta \alpha_{t} . \widehat{L}_{t}$, it is seen that

$$
\sum_{t} \alpha_{t} \cdot h\left(\widehat{L_{t} \cap N}\right)+\sum_{t} \alpha_{t} \cdot h\left(\widehat{L_{t} \backslash N}\right)=\sum_{t} \delta \alpha_{t} \cdot\left(\widehat{L_{t} \cap N}\right)+\sum_{t} \delta \alpha_{t} \cdot\left(\widehat{L_{t} \backslash N}\right)
$$

For each $x \in H, h x \in \tilde{H}$ if and only if $x \in N$. Hence

$$
\sum_{t} \alpha_{t} \cdot h\left(\widehat{L_{t} \cap N}\right)=\sum_{t} \delta \alpha_{t} \cdot\left(\widehat{L_{t} \cap N}\right) .
$$

This implies that $h \tilde{e} \in K \tilde{e}$. Hence $K \tilde{H} \tilde{e} \leqq K \tilde{e}$ and so $K \tilde{H} \tilde{e}=K \tilde{e}$. Therefore $[K \tilde{H} \tilde{e}: K]=1$.

Consequently, $\tilde{h}_{i}=1$ for all $i$. This shows that every irreducible $K \tilde{H}$-module is of $K$-dimension one, and so $\tilde{H}$ is abelian. Hence $N$ is abelian since $N=\tilde{H} \times P$. This completes the proof.

Remark 1. Assume $|P|=2$ or 1 if $p=2$, and assume $|P|=1$ if $p$ is odd. In this case, from [10, Theorem] and the proof of Theorem $1, J(K G)^{2}=0$, and so $J(K G)$ is commutative. Since $G$ is a $p$-nilpotent group with an abelian $p$-Sylow subgroup $P$, by the proof of Theorem1, $N_{G}(P)=C_{G}(P)$. But $N_{G}(P)$ is nonabelian in general. Indeed, if we set that $H$ is a nonabelian finite group of order prime to $p$ and that $G=H \times P$, then $G=N_{G}(P)=$ $C_{G}(P)$ and $N_{G}(P)$ is nonabelian.

Next, we shall have the following main theorem of this paper. This gives a grouptheoretical condition of $G$ for $J(K G)$ to be commutative. 
THEOREM 2. For an arbitrary prime number $p, J(K G)$ is commutative if and only if $G$ is a group of the following two types:

(i) $|G|$ is not divisible by $2^{2}$ when $p=2$, and $|G|$ is not divisible by $p$ when $p$ is odd.

(ii) $G$ is a p-nilpotent group with an abelian p-Sylow subgroup $P . b_{0}=\left|O_{p^{\prime}}(G): G^{\prime}\right|$, $b_{1}=\ldots=b_{a-2}=0$, and if $p$ is odd, $b_{a-1}=0$, where $|P|=p^{a}$ and $b_{k}$ is the number of $p$-regular conjugate classes $K_{j}$ of $G$ such that $\left|K_{j}\right|$ is divisible by $p^{k}$ and not by $p^{k+1}$ for $k=0, \ldots, a$.

Proof. From the proof of Theorem 1 we can assume that $K$ is algebraically closed. So we use notations $n, U_{i}, F_{i}, u_{i}$ and $f_{i}$ as in the proof of Theorem 1 . Put $H=O_{p^{\prime}}(G)$.

Suppose that $J(K G)$ is commutative and that $|G|$ is divisible by $2^{2}$ when $p=2$, and is divisible by $p$ when $p$ is odd. By [11, Theorem 2], $G$ is a $p$-nilpotent group with an abelian $p$-Sylow subgroup $P$. From the proof of Theorem 1, the number of blocks of $K G$ with defect $a$ is equal to the number of nonisomorphic irreducible $K G$-modules $F_{i}$ such that $f_{\mathrm{i}}=1$. Thus, by [1, Theorem 2] and $\left[1\right.$, p. 588], $b_{0}=\left|G: G^{\prime} P\right|=\left|O_{\mathrm{p}}(G): G^{\prime}\right|$. Since $G$ is $p$-nilpotent, we use notations $B_{i}, P_{i}, h_{i}$ and $t_{i}$ as in the proof of Theorem 1. Let $C=\left(c_{i i}\right)_{1 \leqq i, i}{ }{ }_{n}$ be the Cartan matrix for $K G$. It follows from [6, Theorem 3] that $f_{i}=h_{i} t_{i}$ and $u_{i}=p^{a} h_{i}=f_{i}\left|P_{i}\right|$, hence $c_{i i}=\left|P_{i}\right|$ and $c_{i i^{\prime}}=0$ if $i \neq i^{\prime}$. Since $p^{a}=t_{i}\left|P_{i}\right|$ and $\left(h_{i}, p\right)=1$, a block $B_{i}$ has defect $d$ if and only if $\left|P_{i}\right|=p^{d}$. Put $\left|P_{i}\right|=p^{d_{i}}$ for each $i$. We say that $B_{i}$ is of type $(a),(b)$ or $(c)$ as in the proof of Theorem 1 . If $B_{i}$ is of type $(a), B_{i}$ has defect 0 since $p^{a}$ divides $u_{i}=f_{i}$. If $B_{i}$ is of type $(b)$, by [11, Lemma 7], $p=2$ and $u_{i}=2 f_{i}$, and so $B_{i}$ has defect 1 since $2^{a}$ divides $u_{i}$. If $B_{i}$ is of type $(c), B_{i}$ has defect $a$ from the proof of Theorem 1. Let $\left\{K_{1}, \ldots, K_{n}\right\}$ be the set of all $p$-regular conjugate classes of $G$ and let $K_{i}$ have $p$-defect $k_{i}$, that is to say, $\left|K_{i}\right|$ is divisible by $p^{a-k_{1}}$ and not by $p^{a-k_{1}+1}$, for each $i$.

Case 1. $p=2$. Since every $d_{i}$ is 0,1 or $a$ and

$$
C=\left[\begin{array}{ccc}
2^{d_{1}} & & 0 \\
0 & \ddots & 2^{d_{n}}
\end{array}\right],
$$

it follows from $[\mathbf{1}, \S 16]$ that every $k_{i}$ is also 0,1 or $a$. This implies that $b_{1}=\ldots=b_{a-2}=0$.

Case 2. $p$ is odd. Since every $d_{i}$ is 0 or $a$, as in Case 1 , every $k_{i}$ is also 0 or $a$. Hence $b_{1}=\ldots=b_{a-2}=b_{a-1}=0$.

Conversely, suppose that (i) or (ii) holds. If (i) holds, by $\left[\mathbf{1 0}\right.$, Theorem], $J(K G)^{2}=0$, and so $J(K G)$ is commutative. So we can assume that (ii) holds. Since $G$ is $p$-nilpotent, we use notations $B_{i}$ and $P_{i}$ as in the proof of Theorem 1. From (ii), [1, Theorem 2] and [1, p. 588] we have that the number of blocks of $K G$ with defect $a$ is equal to the number of nonisomorphic irreducible $K G$-modules $F_{i}$ such that $f_{i}=1$. This shows that for a block $B_{i}$, $B_{i}$ has defect $a$ if and only if $f_{i}=1$. From the proof of Theorem $1, B_{i} \cong\left(K P_{i}\right)_{f_{i}}$ for each $i$. If $B_{i}$ has defect $0, J\left(B_{i}\right)=0$. If $p=2$ and $B_{i}$ has defect $1,\left|P_{i}\right|=2$ and so $J\left(B_{i}\right)^{2} \cong\left(J\left(K P_{i}\right)^{2}\right)_{f_{i}}=0$ from [10, Theorem]. If $B_{i}$ has defect $a, f_{i}=1$ and hence $J\left(B_{i}\right) \cong$ $J(K P)$.

Case 1. $p=2$. From (ii) every block $B_{i}$ of $K G$ has defect 0,1 or $a$. Hence $J(K G)$ is commutative. 
Case 2. $p$ is odd. By (ii), every block $B_{i}$ of $K G$ has defect 0 or $a$, and so $J(K G)$ is commutative. This finishes the proof of Theorem 2.

Remark 2. When $p$ is odd, by [11, Theorem 1] (cf. [3, Corollary 5.2]), $J(K G)$ is commutative if and only if $J(K G) \subseteq Z(K G)$. But when $p=2$, this does not hold in general. We can assume that $K$ is algebraically closed from the proof of Theorem 1 . Though $J(K G)$ is commutative, $J(K G) \nsubseteq Z(K G)$ if $a \geqq 2$ and there exists a block $B_{i}$ of $K G$ of type (b) (cf. the proof of Theorem 1). Indeed, suppose that $a \geqq 2, J(K G)$ is commutative and there is a block $B_{i}$ of $K G$ of type $(b)$. By [11, Lemma 7], $p=2$. Since $J(K G)$ is commutative and $a \geqq 2$, we use notations $n, m, B_{i}$ alıd $P_{i}$ as in the proof of Theorem 1 . We can write $J(K G)=\sum_{i=1}^{n} \oplus J\left(B_{i}\right)$. Since there is a block $B_{i}$ of type $(b), \sum_{i=m+1}^{n} \oplus J\left(B_{i}\right) \neq 0$. It follows from the proof of Theorem 2 that $m=\left|G: G^{\prime} P\right|$. If $B_{i}$ is of type (c), by the proofs of Theorems 1 and 2 , it is seen that $J\left(B_{i}\right) \cong J(K P)$, and so $\left[J\left(B_{i}\right): K\right]=2^{a}-1$. Thus $[J(K G): K]=\sum_{i=1}^{m}\left[J\left(B_{i}\right): K\right]+\sum_{i=m+1}^{n}\left[J\left(B_{i}\right): K\right]>m\left(2^{a}-1\right)=\left|G: G^{\prime} P\right|\left(2^{a}-1\right)$. Hence $\left[J\left(K\left(G^{\prime} P\right)\right): K\right]>2^{a}-1$ by the proof of [11, Theorem 1]. Therefore $J(K G) \nsubseteq Z(K G)$ by [8, Theorem 2] and [9, Theorem].

An example of the above case is as follows.

EXAMPLE. Assume that $K$ is algebraically closed and $p=2$. Put $G=\langle x, y| x^{4}=y^{3}=1$, $\left.x^{-1} y x=y^{2}\right\rangle . G$ is a 2-nilpotent group with a cyclic 2-Sylow subgroup $P=\langle x\rangle$. The decomposition matrix $D$ for $G$ and the Cartan matrix $C$ for $K G$ are given as

$$
D=\left[\begin{array}{ll}
1 & 0 \\
1 & 0 \\
1 & 0 \\
1 & 0 \\
0 & 1 \\
0 & 1
\end{array}\right], \quad C=\left[\begin{array}{ll}
4 & 0 \\
0 & 2
\end{array}\right] .
$$

We use notations $U_{i}, f_{i}, B_{i}$ and $P_{i}$ as in the proof of Theorem 1. From the proof of Theorem 2, $\left|P_{1}\right|=4$ and $\left|P_{2}\right|=2$. Hence $B_{1} \cong K P$ and $B_{2} \cong\left(K P_{2}\right)_{2}$. This shows that $J(K G)$ is commutative by [10, Theorem]. On the other hand, we have that $f_{1}=1, f_{2}=2$, $K G \cong U_{1} \oplus U_{2} \oplus U_{2}, B_{1}$ is of type $(c)$ and $B_{2}$ is of type $(b)$. Since $y^{-1} x^{-1} y x=y$ and $G / O_{2^{\prime}}(G) \cong P$, it follows that $O_{2^{\prime}}(G)=G^{\prime}$, and so $G^{\prime} P=G$. Hence $\left[J\left(K\left(G^{\prime} P\right)\right): K\right]=$ $[J(K G): K]=|G|-\left(f_{1}^{2}+f_{2}^{2}\right)=7>3=2^{2}-1$. Thus $J(K G) \nsubseteq Z(K G)$ by [8, Theorem 2$]$ and [9, Theorem]. Indeed, $\left\{(1+x) e,\left(1+x^{2}\right) e,\left(1+x^{3}\right) e, 1+x^{2}, x\left(1+x^{2}\right), x\left(1+x^{2}\right) y,\left(1+x^{2}\right) y^{2}\right\}$ is a $K$-basis of $J(K G)$, where $e=1+y+y^{2}$. Using this we have that $J(K G)$ is commutative but $J(K G) \nsubseteq Z(K G)$ since $\left\{x\left(1+x^{2}\right)\right\} y \neq y\left\{x\left(1+x^{2}\right)\right\}$.

\section{REFERENCES}

1. R. Brauer and C. Nesbitt, On the modular characters of groups, Ann. of Math. 42 (1941), $556-590$. 
2. L. Dornhoff, Group representation theory (part B), (Dekker, 1972).

3. W. Hamernik, Group structure and properties of block ideals of the group algebra, Glasgow Math. J. 16 (1975), 22-28.

4. G. O. Michler, Blocks and centers of group algebras, in Lectures on rings and modules, Lecture Notes in Mathematics 246 (Springer-Verlag, 1972), 429-563.

5. K. Morita, On group rings over a modular field which possess radicals expressible as principal ideals, Sci. Rep. of Tokyo Bunrika Daigaku A 4 (1951), 177-194. $1-9$.

6. M Osima, On primary decomposable group rings, Proc. Phys.-Math. Soc. Japan 24 (1942), 409-437.

7. D. S. Passman, Radicals of twisted group rings, Proc. London Math. Soc. (3) 20 (1970),

8. D. A. R. Wallace, Note on the radical of a group algebra, Proc. Cambridge Philos. Soc. 54 (1958), 128-130.

9. D. A. R. Wallace, Group algebras with central radicals, Proc. Glasgow Math. Assoc. 5 (1962), 103-108.

10. D. A. R. Wallace, Group algebras with radicals of square zero, Proc. Glasgow Math. Assoc. 5 (1962), 158-159.

11. D. A. R. Wallace, On the commutativity of the radical of a group algebra, Proc. Glasgow Math. Assoc. 7 (1965), 1-8.

Department of Mathematics

TSUKUBA UNIVERSITY

SAKURA-MURA, IBARAKI

300-31, JAPAN 